EPJ Web of Conferences 66, 03037 (2014)

DOI: $10.1051 /$ epjconf/ 20146603037

(C) Owned by the authors, published by EDP Sciences, 2014

\title{
Mass-angle distributions
}

\section{Insights into the dynamics of heavy element formation}

\author{
D.J. Hinde ${ }^{1, a}$, R. du Rietz ${ }^{1,2}$, E. Williams ${ }^{1}$, C. Simenel ${ }^{1}$, C.J. Lin ${ }^{1,3}$, A. Wakhle ${ }^{1}$, K.J. Cook ${ }^{1}$, M. \\ Dasgupta $^{1}$, M. Evers ${ }^{1}$, and D.H. Luong ${ }^{1}$ \\ ${ }^{1}$ Department of Nuclear Physics, RSPE, Australian National University, ACT 0200, Australia \\ ${ }^{2}$ Current address: Malmö University, Faculty of Technology and Society, 20506 Malmö, Sweden \\ ${ }^{3}$ Current address: China Institute of Atomic Energy, P. O. Box 275(10), Beijing 102413, China.
}

\begin{abstract}
Mass-angle distributions carry detailed information on the characteristics of quasifission, and thus of the dynamics of heavy element formation reactions. Recent experimental results are presented and discussed.
\end{abstract}

\section{Introduction}

When forming heavy elements through heavy-ion fusion reactions, their yield is generally significantly suppressed [1] by quasifission [2]. This is a dynamical non-equilibrium process that results when the combined system formed after capture breaks apart before a compact compound nucleus is formed. Understanding the competition between quasifission and fusion is important for predicting the best reactions to use to form new isotopes of heavy and super-heavy elements (SHE) by fusion.

The probability of quasifission, and the characteristics of the quasifission products, are determined by the diffusive motion over a multi-dimensional potential energy surface (PES). This results either in a compact shape (fusion) or an elongating di-nuclear shape leading to quasifission. The PES describes the energy of the system as a function of important nuclear/di-nuclear shape degrees of freedom. These include overall elongation and mass-asymmetry, and the deformation of and necking between the nascent fragments. The main characteristics of the PES will depend on the overall balance between the repulsive Coulomb and attractive nuclear forces. In addition, nuclear structure causes shapedependent modulations of the smooth liquid drop PES. These can have a large effect, most notably resulting in the well-known mass-asymmetric fission of the actinide elements.

The initial injection point into the PES is an important variable, and depends on entrance-channel mass (charge) asymmetry and deformation alignment [3-5]. The dynamical characteristics that control the motion of the system from the contact point over the PES are the most complex and least understood aspect of the problem, as they are determined by the dissipation and inertia tensors, which can both be affected by shell structure. Since all these effect of shell structure are damped with excitation energy $\left(\mathrm{E}_{x}\right)$, it is expected that there will be a dynamical coupling between these variables and the excitation energy $[6,7]$.

Because of the complex dependence of quasifission characteristics on many variables, and the overlap between quasifission and fusion-fission events in experiments, quasifission is not yet fully

a e-mail: david.hinde@anu.edu.au

This is an Open Access article distributed under the terms of the Creative Commons Attribution License 2.0, which permits unrestricted use, distribution, and reproduction in any medium, provided the original work is properly cited. 
understood. As a wholly dynamical process, a key quantity characterizing quasifission is its timescale - that is the "sticking time" between capture and breakup (scission). Measurements of quasifission mass-angle distributions (MAD) at GSI in the 1980s [2,8] showed that timescales could often be shorter than the rotation time of $\sim 10^{-20} \mathrm{~s}$, and were thus significantly shorter than the typical timescale of fusion fission. The measurement of MAD thus offers a key insight into the quasifission process. Only a few measurements were subsequently made $[9,10]$ until a program of measurements was started at the Australian National University, which is showing a rich variety of phenomena in quasifission. Some recent results are shown in the following sections.

\section{Principle behind the mass-angle distribution}

The measurement of the full range of mass-splits between projectile and target over a wide range of scattering angles results in a two-dimensional matrix, which we refer to as a mass-angle distribution. The relationship of the MAD to the "sticking time" between capture and scission is illustrated schematically in Fig. 1. The projectile nucleus (blue) is incident from the top of the page, and sticks to the larger target nucleus (red). The system then rotates, Fig. 1(b) illustrating schematically angle against time (in arbitrary units) for a single angular momentum value; in a real reaction a distribution will be present. For a parabolic potential, mass-symmetry is approached with an expected time dependence $1-\exp \left(t \tau_{e q}\right)$, where $\tau_{e q}$ is the mass-equilibration time constant [2]. This dependence is sketched in Fig. 1(c).
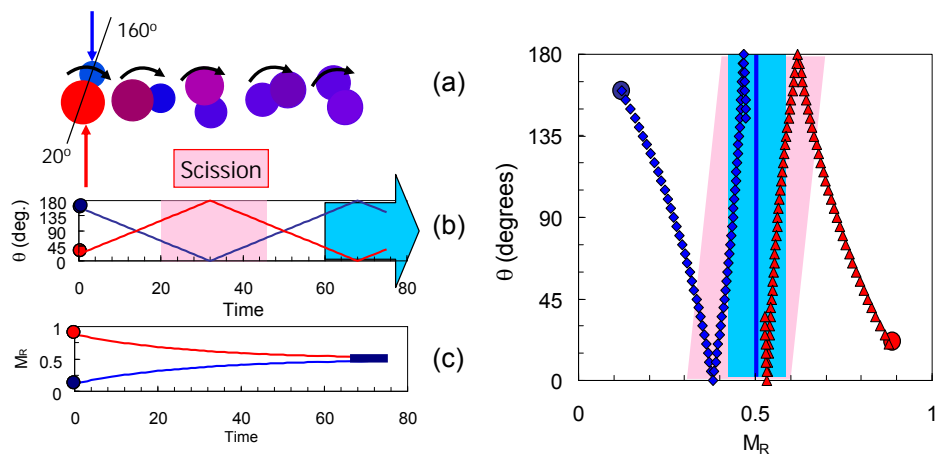

Figure 1. Diagram of the relationship between sticking time and the mass-angle distribution. For the reaction time sequence sketched in (a), the time dependence (arbitrary units) of angle (b) and mass-ratio (c) are shown. Time is not measured directly, but angle and mass-ratio are. The individual mass and angle dependencies combine to give a trajectory (d) on the MAD for a single impact parameter. Including a range of impact parameters, scission after half a turn (pink) or a full turn (blue) will give strong or weak mass-angle correlations respectively.

If scission happens soon after initial contact, then little mass change can occur, and a projectilelike fragment is ejected with mass-ratio $M_{R}$ at backward angle $\theta_{c . m .} . M_{R}$ is defined as the mass of one fragment divided by the total mass involved in the collision. Its complementary binary fragmentation partner with mass-ratio $\left(1-M_{R}\right)$ is found at $\left(\pi-\theta_{c . m .}\right)$, a forward angle. A longer sticking time of the system results in larger rotation angles, and also allows more mass exchange. This evolution is illustrated on the MAD shown in Fig. 1(d). Rotation of the system by $\sim 180^{\circ}$ still results in a substantial mass-angle correlation (pale pink shading in Fig. 1(b),(d)), whilst once the system has turned $\sim 360^{\circ}$ or more, the correlation between the mass ratio and fragment emission angle is washed out, resulting in symmetric mass splits on average, independent of angle (blue shading). 


\section{Extracting reaction times from mass-angle distributions}

Mass angle distributions were measured for the reactions of ${ }^{34} \mathrm{~S}+{ }^{186} \mathrm{~W},{ }^{48} \mathrm{Ti}+{ }^{186} \mathrm{~W}$ and ${ }^{64} \mathrm{Ni}+{ }^{184} \mathrm{~W}$, for a range of beam energies [11], to compare with crystal blocking measurements for reactions of the same elements. The latter were interpreted as giving mean quasifission times of $\sim 10^{-18} \mathrm{~s}$ for all reactions [12]. The measured MAD are shown in Fig. 2. The lower panels show the $\mathrm{M}_{R}$ projections for $45^{\circ} \leq \theta_{c . m} \leq 135^{\circ}$. They show marked differences between the three reactions. The ${ }^{64} \mathrm{Ni}$ reaction gives a minimum in yield at symmetry $\left(\mathrm{M}_{R}=0.5\right),{ }^{48} \mathrm{Ti}$ a broad peak around symmetry, whilst ${ }^{34} \mathrm{~S}$ gives a narrow peak. Together with the correlation of mass and angle seen in the MAD, quasifission with short average reaction times appears dominant in the ${ }^{64} \mathrm{Ni}$ and ${ }^{48} \mathrm{Ti}$ reactions. To obtain more quantitative reaction timescales for the dominant quasifission, a classical Monte Carlo MAD simulation was developed [11].
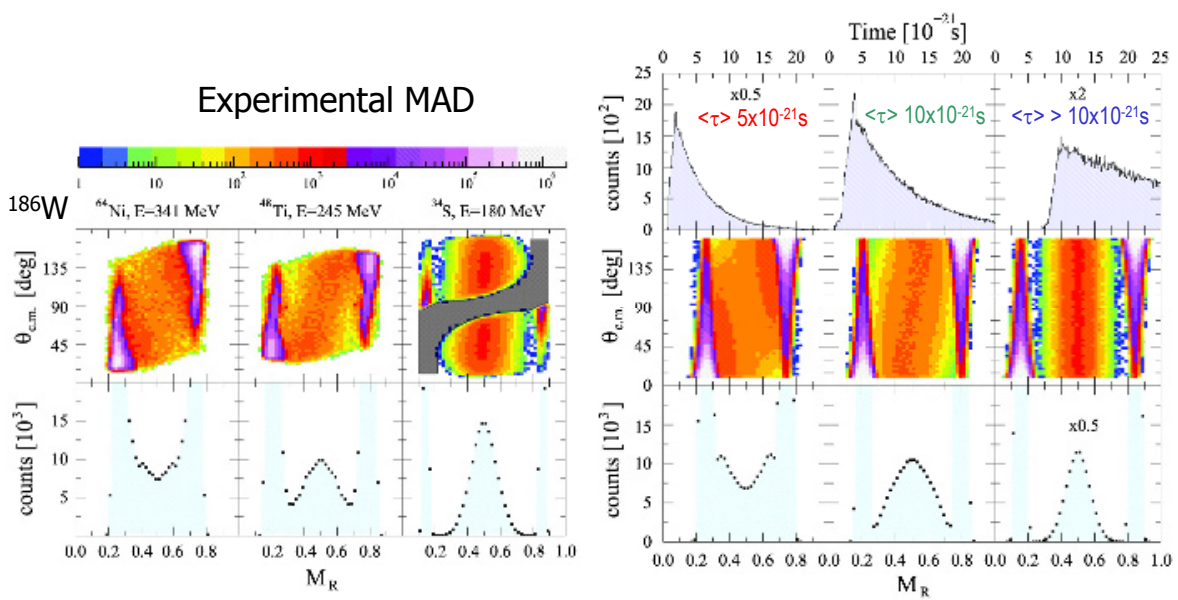

Figure 2. The left panels show the experimental MAD and corresponding projections onto $M_{R}$ for ${ }^{64} \mathrm{Ni}+{ }^{184} \mathrm{~W}$, ${ }^{48} \mathrm{Ti}+{ }^{186} \mathrm{~W}$ and ${ }^{34} \mathrm{~S}+{ }^{186} \mathrm{~W}$ (see text). The right panels show simulated MAD and $M_{R}$ spectra for the same reactions and energies, with the sticking time distributions (above) which give good agreement between the simulations and the measurements.

Using the time distributions shown in the top right panels of Fig. 2, the simulated MAD (shown below them) reproduce the features of the experimental MAD. The corresponding $M_{R}$ spectra for $45^{\circ} \leq \theta_{c . m} . \leq 135^{\circ}$ (lowest right-hand panels of Fig. 2) also agree. The mean scission time for quasifission is quite well defined for these reactions, at $5 \times 10^{-21} \mathrm{~s}$ for ${ }^{64} \mathrm{Ni}$, and $10 \times 10^{-21} \mathrm{~s}$ for ${ }^{48} \mathrm{Ti}$. For the ${ }^{34} \mathrm{~S}$ reaction, the reaction times (rotation angles) are too large to conclude more than that only a few events occur before $10 \times 10^{-21} \mathrm{~s}$. These results, when compared with the longer crystal blocking times, suggest the need for a consistent assessment of all experimental techniques for determination of fission times, including pre-fission neutrons [13-16]. A coordinated series of measurements for the same reactions, using all the different time-sensitive techniques, would give valuable insights.

\section{Isolating effects of entrance-channel mass-asymmetry}

It is expected that the charge product in the entrance channel $\left(Z_{1} Z_{2}\right)$ is a key predictor of the inhibition of fusion through faster processes such as quasifission or deep-inelastic collisions. The fission MAD 


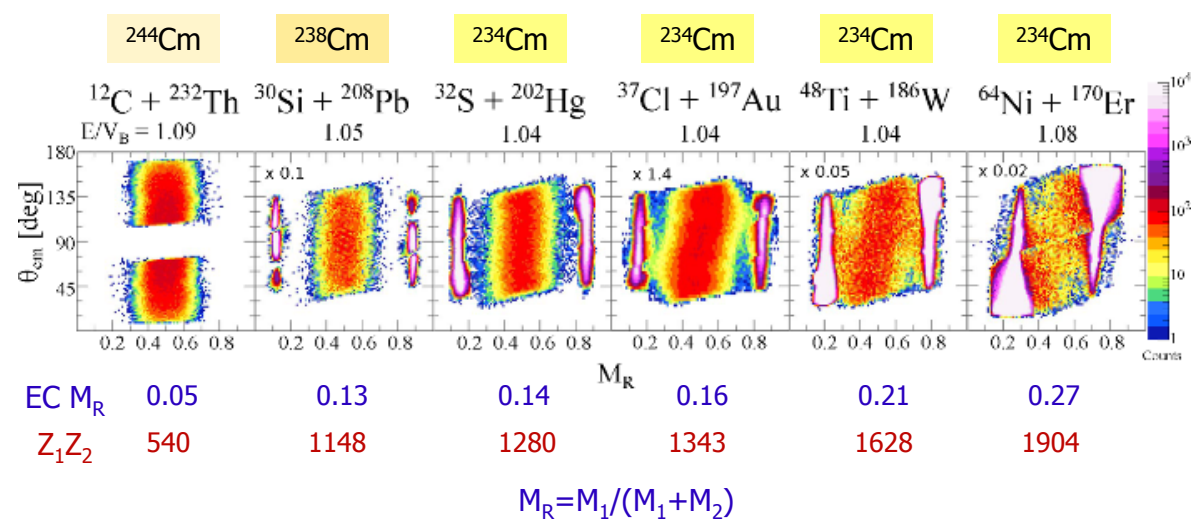

Figure 3. Measured mass-angle distributions for reactions forming isotopes of Curium at energies $\mathrm{E}$ above the respective capture barriers $\mathrm{V}_{B}$. The $\mathrm{x}$ factor multiplies the maximum counts in the logarithmic colour scale. For ${ }^{234} \mathrm{Cm}$ formed by projectiles from ${ }^{32} \mathrm{~S}$ to ${ }^{64} \mathrm{Ni}$, with entrance channel mass-ratio $\left(\mathrm{EC}_{R}\right)$ varying from 0.14 to 0.27 , the $\mathrm{MAD}$ and thus the mean reaction timescale changes significantly.

would also be expected to change with increasing charge product (or the related entrance-channel mass-asymmetry), reflecting the increasing probability of fast non-equilibrium processes that reduce the probability of forming a compact compound nucleus (fusion). Thus where quasifission occurs in less than one rotation, the MAD would be expected to show a changing mass-angle correlation with increasing charge product, already seen in the previous section. To simplify the situation, comparing different reactions forming the same compound nucleus reduces the number of unknowns, as the PES is the same for all reactions, and only the injection points into the PES differ.

Experimental MAD for reactions forming isotopes of $\mathrm{Cm}(\mathrm{Z}=96)$ are presented in Fig. 3, for which the entrance channel $\mathrm{M}_{R}$ and $\mathrm{Z}_{1} \mathrm{Z}_{2}$ value are given. The beam energies are all above-barrier, to reduce sensitivity to possible shell effects (see next section). The four reactions forming ${ }^{234} \mathrm{Cm}$ ( which are those with the heaviest projectiles) have charge products from 1280 to 1904. They show very similar behaviour to the different projectiles bombarding $\mathrm{W}$, namely a transition from no massangle correlation with ${ }^{32} \mathrm{~S}$ (timescales longer than $\sim 10^{-20} \mathrm{~s}$ ) to a few $10^{-21} \mathrm{~s}$ for ${ }^{64} \mathrm{Ni}$, where there appears to be a continuum from deep-inelastic to quasifission, unlike the reactions with the lighter projectiles. The results from the measurements shown in Figs. 2,3 might suggest that quasifission competition is simply dependent on charge product. When we investigate MAD at energies below the capture barrier, in more gentle collisions, this proves to be far from the truth.

\section{Effect of closed shells and N/Z asymmetry in the entrance channel}

To investigate the effect on quasifission of closed shells in the entrance channel, measurements [17] of MAD were made for ${ }^{40,44,48} \mathrm{Ca}$ projectiles bombarding targets of ${ }^{208,204} \mathrm{~Pb}$ (forming ${ }^{248,252}$ No with $\left.\mathrm{Z}_{C . N .}=102\right)$, and for ${ }^{48} \mathrm{Ti}$ bombarding ${ }^{200} \mathrm{Hg}\left({ }^{248} \mathrm{No}\right)$ and ${ }^{208} \mathrm{~Pb}\left({ }^{256} \mathrm{Db}\right)$. Measurements were made a few percent below the average fusion barrier energy to ensure low relative velocity at contact. The MAD and projected $\mathrm{M}_{R}$ spectra are shown in Fig. 4, together with reference data for the ${ }^{16} \mathrm{O}+{ }^{238} \mathrm{U}$ reaction at an above-barrier energy, forming ${ }^{254} \mathrm{Fm}$. The Gaussian fit standard deviations $\sigma_{M R}$, with experimental uncertainties, and the $\sigma_{M R}$ value for ${ }^{48} \mathrm{Ca}+{ }^{208} \mathrm{~Pb}$ from Ref. [18] are tabulated. Despite having similar or identical $\mathrm{Z}_{1} \mathrm{Z}_{2}$, and forming similar or identical compound nuclei, there is a 
INPC 2013

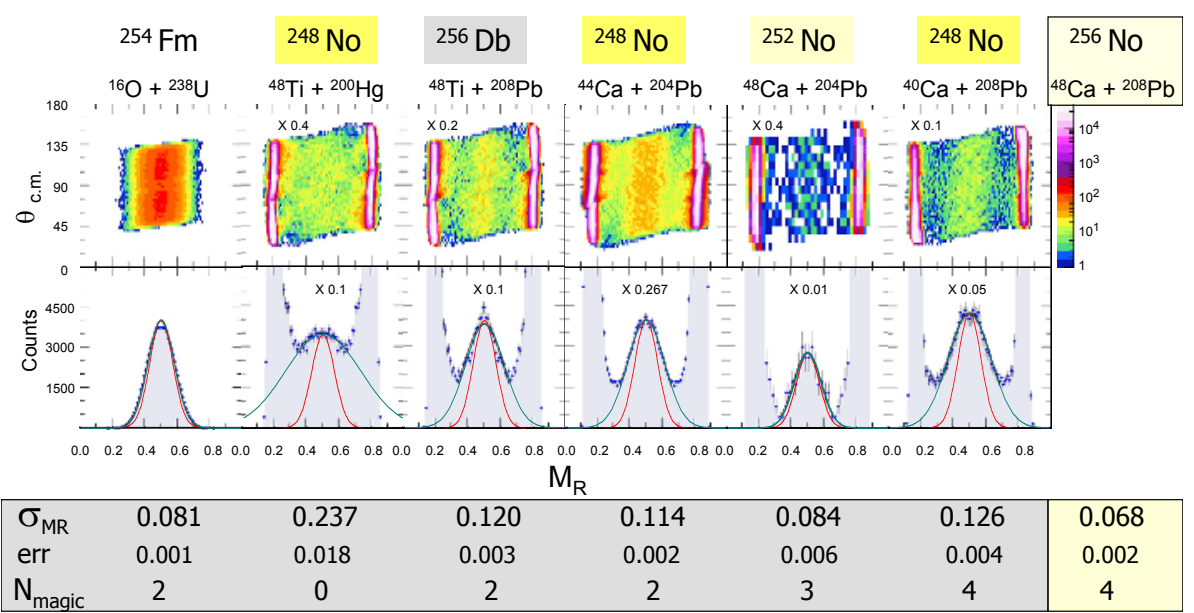

Figure 4. Measured mass-angle distributions for each reaction (upper panels). The factor multiplies the maximum counts of the logarithmic color scale (right). In the projected mass ratio spectra (lower panels) the scale factor multiplies the counts scale on the left. Gaussian fits to the region around $M_{R}=0.5$ are shown (turquoise lines), whose standard deviations $\sigma_{M R}$ are given below. Gaussian functions with $\sigma_{M R}=0.07$ (thin red lines) are shown for reference.

wide variation in the $\sigma_{M R}$ values, indicating a significant variation in the probability/characteristics of quasifission. To understand this, Fig. 5 shows $\sigma_{M R}$ as a function of the number of magic numbers $\mathrm{N}_{m}$ in the entrance channel. There is a strong correlation, with $\sigma_{M R}$ approaching the expectation for fusion-fission $\left(\sigma_{M R}=0.07\right)$ with increasing $\mathrm{N}_{m}$. This suggests that reactions involving nuclei having several magic numbers form a true compact compound nucleus with higher probability. It seems likely that this is associated with reduced energy dissipation as the two nuclei overlap, allowing more compact shapes to be reached. Supporting this hypothesis, systematic analysis of xn evaporation residue cross sections in reactions forming Th isotopes has suggested enhanced fusion probabilities for two magic numbers in the entrance channel [19].

It is notable that the ${ }^{40} \mathrm{Ca}+{ }^{208} \mathrm{~Pb}$ reaction, which has maximal magicity, has a large $\sigma_{M R}$. Only this reaction has a large N/Z asymmetry. Protons and neutrons will tend to be transferred early in the reaction to equalize N/Z. TDHF calculations [17] of nucleon exchange prior to capture confirm this. Using a particle number projection technique [20], the probability of no transfer was calculated to be only $\sim 10^{-4}$, the most probable collision partners at the moment of capture being ${ }^{42} \mathrm{Ar}$ and ${ }^{206} \mathrm{Po}$, which have $\mathrm{N}_{m}=0$. In contrast, the ${ }^{48} \mathrm{Ca}+{ }^{208} \mathrm{~Pb}$ reaction (where the $\mathrm{N} / \mathrm{Z}$ values of the two nuclei are similar) is calculated to remain in its initial mass and charge partition with probability $\sim 0.5$, thus largely retaining the initial magicity. Thus matching of N/Z in the entrance channel should be an important condition in the enhancement of fusion in reactions of magic (and especially doubly-magic) nuclei.

\section{Conclusions}

Mass-angle distributions give unique insights into the dynamics and timescale of quasifission. In collisions at energies well-above the capture barrier, the charge product is the key indicator of the probability and characteristics of quasifission. However, new evidence shows that in gentle collisions, the presence of several magic numbers in the two colliding nuclei makes it easier for the system to 


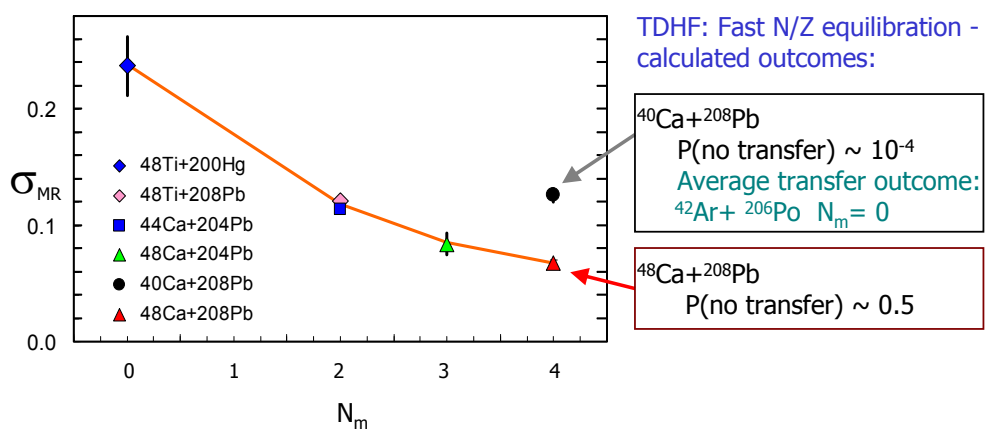

Figure 5. (Left) Standard deviations $\sigma_{M R}$ of the fission-like mass distributions as a function of the number $N_{m}$ of magic numbers in the entrance channel. The line guides the eye. The expectations of $\sigma_{M R}$ for fusion-fission are in the range 0.06 to 0.08 . (Right) TDHF calculation outcomes are summarized (see text).

reach compact shapes, and thus undergo true fusion, with a concomitant increase expected in ER cross sections. This effect is substantially attenuated if the N/Z ratios of the two colliding nuclei are significantly mismatched. This might have important implications for eventual use of radioactive beams to form new isotopes of heavy elements.

This work was supported by the Australian Research Council Discovery Grant DP110102858, ARC Laureate Fellowship FL110100098 (MD), and ARC Future Fellowship FT120100760 (CS).

\section{References}

[1] D.J. Hinde et al., Phys. Rev. Lett. 189, 282701 (2002)

[2] J. Tōke et al., Nuclear Physics A440, 327 (1985)

[3] D.J. Hinde et al., Phys. Rev. C 53, 1290 (1996)

[4] R. Rafiei et al., Phys. Rev. C 77, 024606 (2008)

[5] C.J. Lin et al., Phys. Rev. C 85, 014611 (2012)

[6] D.J. Hinde et al., Phys. Rev. Lett. 101, 092701 (2008)

[7] P. Armbruster, Annu. Rev. Nucl. Part. Sci. 50, 411 (1990)

[8] W.Q. Shen et al., Phys. Rev. C 36, 115 (1987)

[9] J. Velkovska et al., Phys. Rev. C 59, 1506 (1999)

[10] B.B. Back et al., Phys. Rev. C 53, 1734 (1996)

[11] R. du Rietz et al., Phys. Rev. Lett. 106, 052701 (2011)

[12] J.U. Andersen et al., Phys. Rev. C 78, 064609 (2008)

[13] D.J. Hinde et al., Phys. Rev. C 45, 1229 (1992)

[14] D.J. Hinde et al., Nucl. Phys. A553, 255c (1993)

[15] D.J. Hinde et al., Phys. Rev. Lett. 52, 986 (1984); ibid. 53, 2275 (1984)

[16] L. Donadille et al., Nucl. Phys. A656, 259 (1999)

[17] C. Simenel et al., Phys. Lett. B 710, 607 (2012)

[18] E. Prokhorova et al., Nucl. Phys. A802, 45 (2008)

[19] D.J. Hinde and M. Dasgupta, Phys. Lett. B 622, 23 (2005)

[20] C. Simenel, Phys. Rev. Lett. 105, 192701 (2010) 